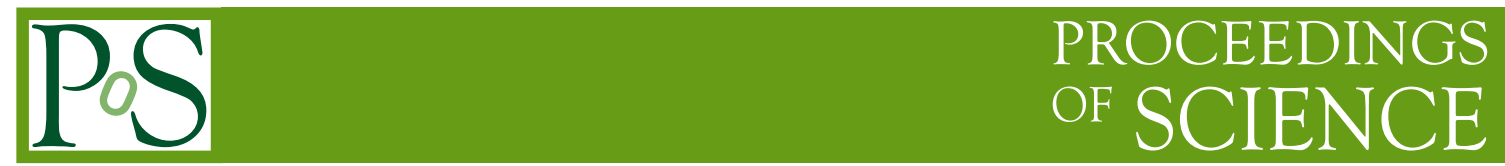

\title{
Scale setting via the $\Omega$ baryon mass
}

\author{
Stefano Capitani ${ }^{1}$, Michele Della Morte ${ }^{1,2}$, Georg von Hippel ${ }^{* 1}$, Bastian \\ Knippschild ${ }^{1}$, Hartmut Wittig ${ }^{1,2}$ \\ ${ }^{1}$ Institut für Kernphysik, Johannes-Gutenberg-Universität Mainz, 55099 Mainz, Germany \\ ${ }^{2}$ Helmholtz-Institut Mainz, University of Mainz, 55099 Mainz, Germany \\ hippel@kph.uni-mainz.de
}

We present the first results of an ongoing effort to determine the lattice scale on the $N_{f}=2$ CLS lattice ensembles via the mass of the $\Omega$ baryon. Results from different methods are compared, and various sources of systematic uncertainty are discussed.

XXIX International Symposium on Lattice Field Theory

July $11-162011$

Squaw Valley, Lake Tahoe, California

${ }^{*}$ Speaker. 


\section{Introduction}

In order to give absolute values for dimensionful quantities (including masses and decay constants) from a lattice simulation, we need to know the lattice spacing $a$.

Since no unique prescription for scale-setting exists, there is a certain ambiguity arising from the choice of the dimensionful quantity used to set the scale, which however is at least formally small since it is of higher order in the lattice spacing. Of more practical importance are the uncertainties that arise from an inaccurate knowledge of the scale caused by statistical and systematic errors on the relevant quantity.

Here, we present the result of our effort to set the scale via the mass of the $\Omega$ baryon [1]. The $\Omega$ mass has a number of advantages over other quantities, in that the $\Omega$ is stable in QCD (as opposed to e.g. the $K^{*}$ meson), and that its mass is only very weakly dependent on the light quark mass (as opposed to e.g. the nucleon). In addition, all calculations necessary to determine the scale from the $\Omega$ mass can be done in the fully relativistic theory (as opposed to e.g. a scale determination from the $\Upsilon 2 \mathrm{~S}-1 \mathrm{~S}$ splitting), and no renormalization constant is needed for $m_{\Omega}$ (as opposed to e.g. $f_{K}$ ). Possible disadvantages of using the $\Omega$ baryon include the less favourable signal-to-noise ratio for baryons, which may render the extraction of a precise value for $a m_{\Omega}$ difficult. In an $N_{\mathrm{f}}=2$ simulation, an additional potential downside of the $\Omega$ baryon is the quenching of the strange quark, which may introduce additional (presumably small) systematic effects.

The $\Omega$ baryon thus provides a way to set the scale that reduces many sources of systematic error in exchange for an increase in statistical error. To the extent that this increased error can be beaten by performing more measurements, this is a rather favourable situation.

\subsection{The CLS ensembles}

Coordinated Lattice Simulations (CLS) is a consortium designed to pool the human and computer resources of several teams in Europe interested in lattice QCD. CLS member teams are located at CERN, in Germany (Berlin, DESY/Zeuthen, Mainz, Wuppertal), Italy (Rome, Milan) and Spain (Madrid, Valencia). All CLS simulations use either M. Lüscher's implementation of the DDHMC algorithm [2] or the MP-HMC algorithm by M. Marinkovic and S. Schaefer [3] to efficiently simulate $N_{f}=2$ Wilson QCD with non-perturbative $\mathrm{O}(a)$ improvement on a variety of computer architectures ranging from $\mathrm{PC}$ clusters to the BlueGene/P at NIC/Forschungszentrum Jülich.

The ensembles used in the present analysis are listed in table 1.

\section{Methods}

\subsection{Measurements}

We measure the two-point correlators for mesons and baryons using Gaussian-smeared sources [4] with HYP-smeared links [5] in the covariant Laplacian to suppress excited state contributions. The parameters of the smearing are fixed at each $\beta$ so as to correspond to a smearing radius of approximately $0.4 \mathrm{fm}$. Multiple point sources per configuration are used in order to improve statistics. For the vector mesons and baryons, we also average over multiple polarizations to further reduce statistical errors. 

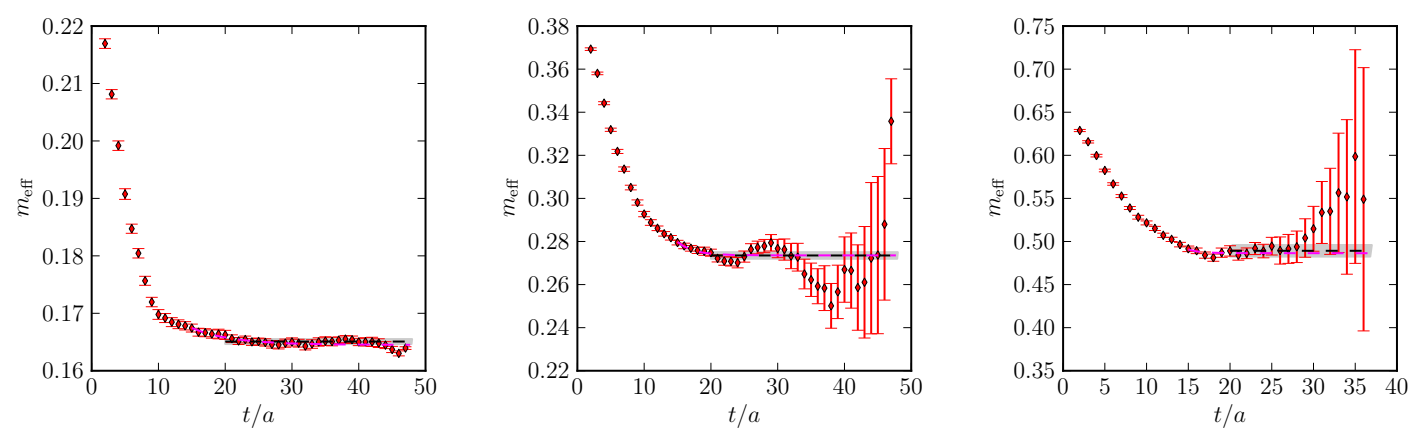

Figure 1: Examples of effective mass plots with plateaux and two-state fits shown for a pseudoscalar and vector meson and a decuplet baryon channel, corresponding to a trial $K, K^{*}$ and $\Omega$ on the $N 4$ ensemble, respectively.

From each measured correlator we extract the effective masses and apply the following methods to extract estimates for the mass of the ground state: First, a naive plateau fit is performed for each correlator, starting at a reasonably large Euclidean time; secondly, taking the plateau value as the starting point, a two-state fit including the leading excited state contribution is performed with a larger time range. Finally, another two-state fit with the same time range is performed for the baryonic channel, in which the gap between the ground and excited state is fixed to the theoretically expected gap of $2 m_{\pi}$ using the measured pion mass. Examples of effective mass plots are shown in fig. 1.

\subsection{Fixing $\kappa_{\text {strange }}$}

In order to establish the physical value of the $\Omega$ baryon, we need to fix the strange quark mass to its physical value. Since we intend to set the scale from the $\Omega$ mass, a scale-free renormalization

\begin{tabular}{lllll}
\hline \hline & $\beta$ & $T \times L^{3}$ & $\kappa$ & $N_{\text {cfg }}$ \\
\hline A3 & 5.2 & $64 \times 32^{3}$ & 0.13580 & 261 \\
A4 & & & 0.13590 & 371 \\
A5a & & & 0.13594 & 201 \\
\hline E5c & 5.3 & $64 \times 32^{3}$ & 0.13625 & 112 \\
F6 & & $96 \times 48^{3}$ & 0.13635 & 192 \\
F7a & & & 0.13638 & 249 \\
\hline N3 & 5.5 & $96 \times 48^{3}$ & 0.13640 & 149 \\
N4 & & & 0.13650 & 142 \\
N5 & & & 0.13660 & 236 \\
O7 & & $128 \times 64^{3}$ & 0.13671 & 78 \\
\hline \hline
\end{tabular}

Table 1: CLS ensembles used in this analysis. $N_{\text {cfg }}$ is the number of configurations used for the calculation of hadron masses in this study, not the total number generated. 


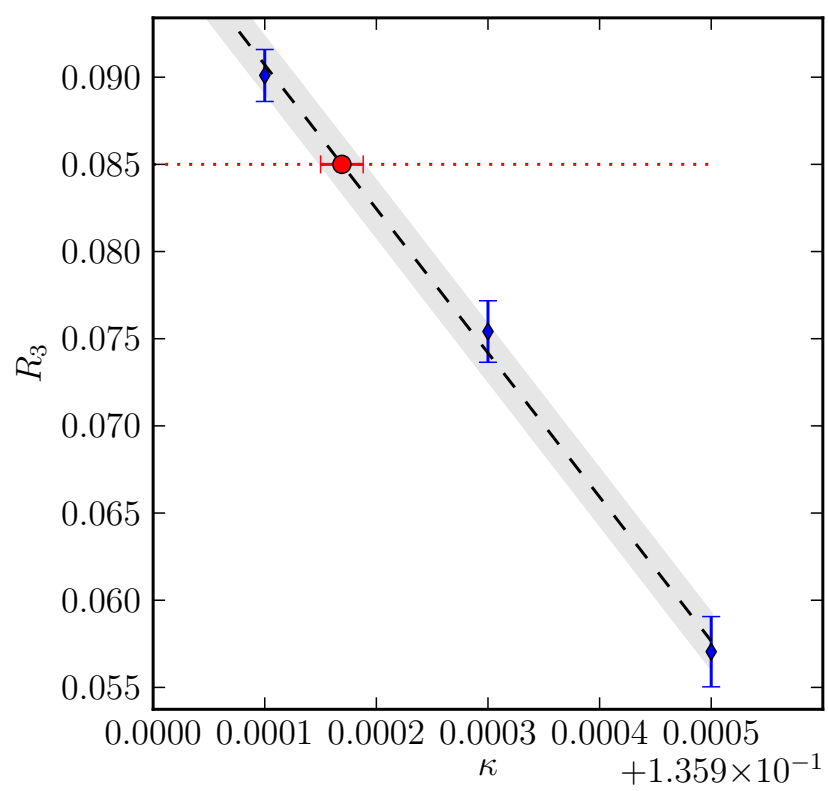

Figure 2: An example of the interpolation to the physical value of the strange quark mass. The measured ratios are shown as blue diamonds, and the interpolated value as the red circle.

condition is needed for this purpose, and we therefore will fix $\kappa_{\text {strange }}$ by demanding that a ratio of masses equals its physical value. Different choices of the ratio used correspond to different renormalization conditions, which will introduce an ambiguity in the scale through the resulting ambiguity in $\kappa_{\text {strange }}$.

The three ratios which we have chosen to fix $\kappa_{\text {strange }}$ are

- $R_{1}=m_{K} / m_{K^{*}}$, which is the ratio originally used by the CERN group in [6],

- a chirally improved variant $R_{2}=\left(m_{K}^{2}-\frac{1}{2} m_{\pi}^{2}\right) / m_{K^{*}}^{2}$, from which the leading $m_{q}$ dependence of $R_{1}$ has been removed, and

- $R_{3}=\left(m_{K}^{2}-\frac{1}{2} m_{\pi}^{2}\right) / m_{\Omega}^{2}$, which is obtained by replacing the $K^{*}$ resonance by the stable $\Omega$ baryon in $R_{2}$.

Determining all three ratios $R_{i}$ requires us to measure $m_{\pi}, m_{\mathrm{PS}}^{\mathrm{qs}}, m_{\mathrm{V}}^{\mathrm{qs}}$ and $m_{\mathbf{1 0}}^{\mathrm{sss}}$ for a range of trial $\kappa_{\text {strange }}$ at each value of $\beta$ and $\kappa_{\text {sea }}$; we then determine the value of $\kappa_{\text {strange }}$ corresponding to the physical strange quark mass by interpolating to the physical value of $R_{i}$. An example is shown in fig. 2.

The difference in the value of $\kappa_{\text {strange }}$ that results from the different mass fits is generally less than the statistical errors, which implies that the systematic errors from excited states are well under control. Similarly, the difference between a linear and a quadratic interpolation in $\kappa_{\text {strange }}$ or in $\kappa_{\text {strange }}^{-1}$ is much smaller than the statistical errors. All statistical errors were determined using the UWerr procedure [7] for error propagation including the effects of autocorrelations. In the following, we have chosen the results from the fit with a fixed gap of $2 m_{\pi}$ as our final value. 

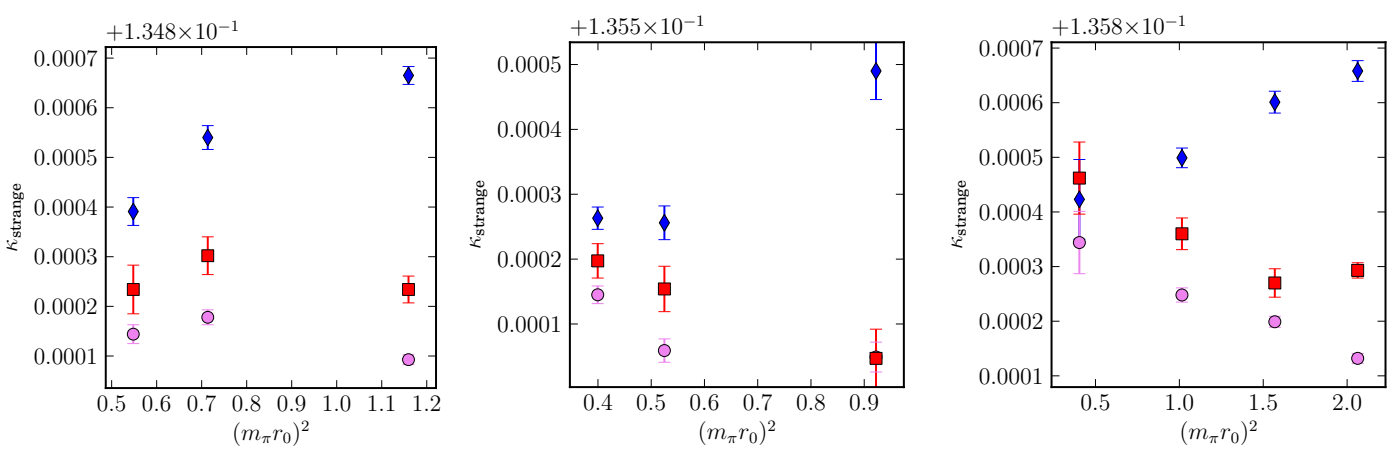

Figure 3: The values of $\kappa_{\text {strange }}$ from the three renormalization conditions studied. From left to right, the $\beta=5.2,5.3$ and 5.5 ensembles are shown with the values obtained from $R_{1}, R_{2}$ and $R_{3}$ plotted as blue diamonds, pink circles and red squares, respectively.

On the other hand, the ambiguity in $\kappa_{\text {strange }}$ from the choice of the renormalization condition is significant, but decreases as expected towards the chiral limit, as can be seen in fig. 3. We find that condition 3, which employs the $\Omega$ baryon and a chirally improved estimate of the strange quark mass, is the most stable and therefore we choose it for our final analysis.

\subsection{Fixing the scale}

The value $r_{0} / a$ of the Sommer scale in lattice units has been determined on the CLS configurations by Knechtli and Leder [9]. On each ensemble, we combine their results for $r_{0} / a$ and our results for $a m_{\Omega}$ using the UWerr procedure [7] in order to take into account both the correlations between observables and the autocorrelations within each ensemble (for an in-depth discussion of the issues associated with the latter cf. [8]).

Since our respective measurements for the $\beta=5.5$ ensembles were performed on non-overlapping subsets, we combine the results on these ensembles neglecting the correlations between $r_{0}$ and $m_{\Omega}$; since we find that the error of $r_{0} m_{\Omega}$ is dominated by the error on $a m_{\Omega}$, any resulting systematic error may be assumed to be small.

The finite combination $r_{0} m_{\Omega}$ can now be extrapolated to the physical point in $a^{2}$ and in $\left(m_{\pi} / m_{\Omega}\right)^{2}$. To estimate systematic uncertainties, we used four different fits:

- a linear fit in $\left(m_{\pi} / m_{\Omega}\right)^{2}$, assuming no lattice artifacts,

- a quadratic polynomial in $\left(m_{\pi} / m_{\Omega}\right)^{2}$, assuming no lattice artifacts,

- a linear fit in $\left(m_{\pi} / m_{\Omega}\right)^{2}$ at each $\beta$ separately, followed by a linear fit of the chirally extrapolated results in $\left(a / r_{0}\right)^{2}$, and

- a combined continuum and chiral extrapolation by a fit linear in both $\left(m_{\pi} / m_{\Omega}\right)^{2}$ and $\left(a / r_{0}\right)^{2}$.

The first two fits, which are made under the assumption that lattice artifacts are absent from the combination $r_{0} m_{\Omega}$, are motivated by the scaling behaviour seen in fig. 4 . All fits agree within their respective errors. 

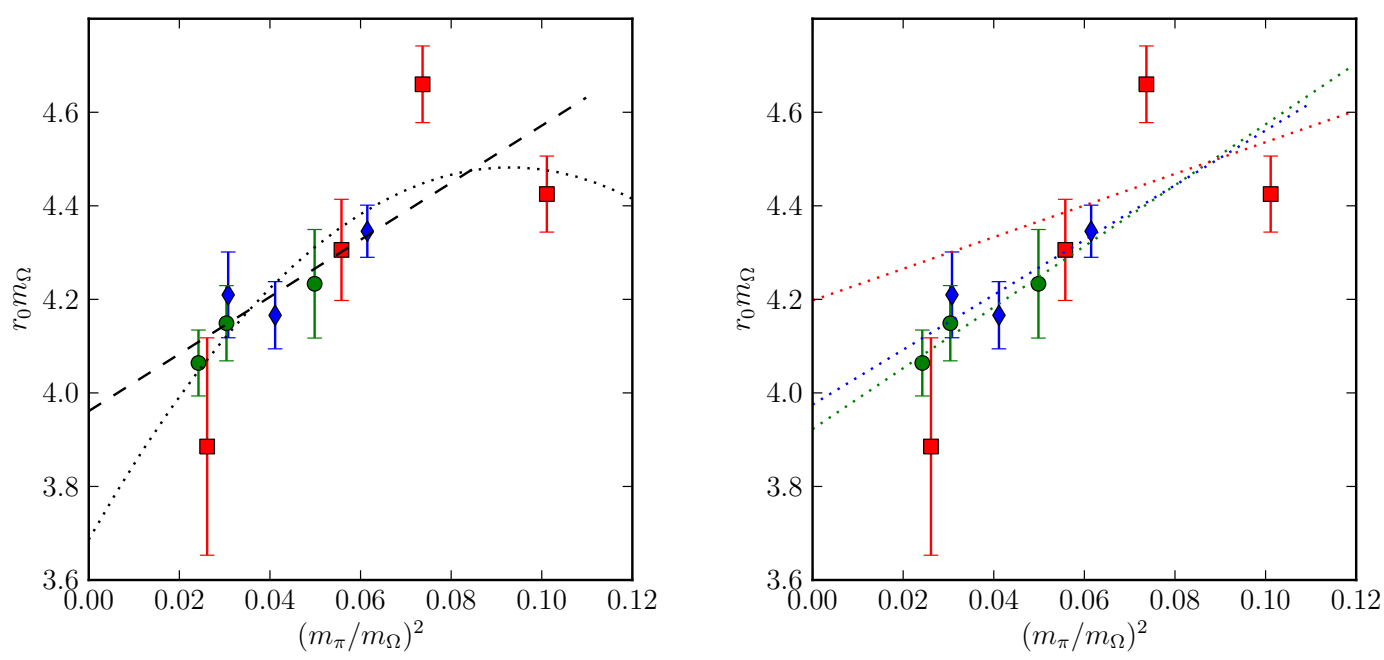

Figure 4: Left: the joint linear and quadratic chiral extrapolations of $r_{0} m_{\Omega}$ ignoring discretization effects; right: the separate chiral extrapolations of $r_{0} m_{\Omega}$ at each value of $\beta$. The $\beta=5.2,5.3$ and 5.5 ensembles are shown as blue diamonds, green circles and red squares, respectively.

\section{Results}

A combined continuum and chiral extrapolation of $r_{0} m_{\Omega}$ gives a physical value of $r_{0} m_{\Omega}=$ 3.99(12)(9), where the errors are statistical and systematic, with the systematic error estimated from the spread of the results obtained using the different fitting strategies both for the mass extraction and in the extrapolation.

Combining this with the experimental value $m_{\Omega}=1672.45(29) \mathrm{MeV}$ [10] gives a value of $r_{0}=0.471(14)(10) \mathrm{fm}$ for the Sommer scale, which is compatible with the ETMC result [11] of $r_{0}=0.465(6)(15)$ using the nucleon.

Performing a separate chiral extrapolation of $a m_{\Omega}$ to the physical pion mass at each value of $\beta$, we find the lattice spacings at the physical point to be

\begin{tabular}{llll}
\hline \hline$\beta$ & 5.2 & 5.3 & 5.5 \\
\hline$a[\mathrm{fm}]$ & $0.079(3)(2)$ & $0.063(2)(2)$ & $0.050(2)(2)$ \\
\hline \hline
\end{tabular}

We note that the lattice spacings obtained from the $\Omega$ baryon mass are significantly finer than those previously obtained [12] for the same ensembles using the CERN method [6]. This is not entirely unexpected, since the CERN method uses an unphysical reference point of $m_{\pi} / m_{K}=0.85$ to make contact with the physical hadron masses, and was intended primarily for determining the relative scale between different $\beta$ values rather than an absolute value of the lattice scale.

The new values presented here are, however, compatible with the results obtained in an entirely separate approach from $f_{K}$ [13], as well as with the results from an independent analysis of the same data using a different error estimator and performing the chiral extrapolation in $\left(m_{\pi} / m_{N}\right)^{2}$ [14], which gives us confidence in the robustness of the results. 


\section{Conclusions}

The mass of the $\Omega$ baryon provides a way to set the scale in a way that is particularly insensitive to the sea quark mass, and independent of renormalization constants and effective theories. We find that the relatively large statistical errors of the $\Omega$ baryon mass can be controlled, and that the systematic errors due to excited states are also well under control; hence the $\Omega$ provides an attractive means to set the scale.

Using $m_{\Omega}$, we have determined the Sommer scale to be $r_{0}=0.471(14)(10) \mathrm{fm}$ and have set the lattice spacing on the $N_{f}=2$ CLS lattices. The lattice spacings determined in this way are compatible with those found in an independent analysis employing $f_{K}$ to set the scale. We find that the lattices are finer than had been previously expected. What influence this will have on the question of finite-volume effects on studies of hadronic structure [15] remains to be seen.

In the immediate future, we intend to increase the number of measurements on the $\mathrm{O} 7 \mathrm{ensem}-$ ble, which will reduce the statistical error on the lattice spacing at $\beta=5.5$; in the longer run, we will also include in our analysis the G8 and N6 ensembles currently being generated, which should help us to improve our control of the chiral extrapolation.

\section{References}

[1] B. Brandt et al., PoS LATTICE2010 (2010) 164 [arXiv:1010.2390].

[2] M. Lüscher, Comput. Phys. Commun. 165 (2005) 199-220. [hep-lat/0409106].

[3] M. Marinkovic, S. Schaefer, PoS LATTICE2010 (2010) 031. [arXiv:1011.0911].

[4] S. Güsken et al., Phys. Lett. B227 (1989) 266.

[5] A. Hasenfratz and F. Knechtli, Phys. Rev. D64 (2001) 034504. [hep-lat/0103029].

[6] L. Del Debbio, L. Giusti, M. Lüscher, R. Petronzio, N. Tantalo, JHEP 0702 (2007) 056. [hep-lat/0610059].

[7] U. Wolff, Comput. Phys. Commun. 156 (2004) 143-153. [hep-lat/0306017].

[8] S. Schaefer, R. Sommer, F. Virotta (ALPHA Collaboration), Nucl. Phys. B 845 (2011) 93. [arXiv: 1009.5228].

[9] F. Knechtli, B. Leder (ALPHA collaboration), in these proceedings.

[10] K. Nakamura et al. (Particle Data Group), J. Phys. G 37 (2010) 075021.

[11] C. Alexandrou et al. (ETM Collaboration), Phys. Rev. D80 (2009) 114503. [arXiv:0910.2419].

[12] S. Capitani, M. Della Morte, E. Endress, A. Jüttner, B. Knippschild, H. Wittig, M. Zambrana, PoS LAT2009 (2009) 095. [arXiv:0910.5578].

[13] M. Marinkovic et al. (ALPHA collaboration), in these proceedings.

[14] B. Knippschild, Ph.D. dissertation, University of Mainz 2011.

[15] B. Brandt et al., Eur.Phys.J. ST 198 (2011) 79-94. [arXiv:1106.1554]. 